\title{
Developments in the use of EISCAT for interplanetary scintillation
}

\author{
R. A. Fallows, A. R. Breen, and G. D. Dorrian \\ Institute of Mathematics and Physics, Aberystwyth University, Penglais, Aberystwyth, SY23 3BZ, Wales, UK \\ Received: 21 December 2007 - Revised: 11 April 2008 - Accepted: 14 April 2008 - Published: 5 August 2008
}

\begin{abstract}
The antennas of EISCAT have been used for interplanetary scintillation (IPS) studies of the solar wind for many years. The main science found from these studies is obtained through the cross-correlation of signals from antennas having the longest baseline, providing more accurate information on the different solar wind streams which may be present in the line of sight. The development of dualfrequency IPS observations between the $1.4 \mathrm{GHz}$ receivers at the remote sites and Troms $\varnothing$, has allowed the use of the EISCAT Svalbard Radar for IPS, increasing the available baselines to the extent that three solar wind streams can sometimes be identified in the cross-correlation functions. A weak-scattering model incorporating three possible solar wind streams and dual observing frequencies is discussed and some results presented. A recent study found that the current sampling bandwidth limits the sensitivity of IPS observations at EISCAT. Methods of increasing the sensitivity, and the results of trials, are discussed.
\end{abstract}

Keywords. Interplanetary physics (Solar wind plasma) Radio science (Radio wave propagation; Signal processing)

\section{Introduction}

Interplanetary scintillation (IPS) arises from the diffraction of radio waves from a distant, compact source by density variations within the solar wind. Measurements of IPS have been used for many years to probe the solar wind throughout the inner heliosphere (e.g. Hewish et al., 1964; Coles, 1978; Manoharan and Ananthakrishnan, 1990; Breen et al., 1996b; Canals et al., 2002).

The three antennas of the European Incoherent SCATter radar (EISCAT) have been used to probe the solar wind at all heliographic latitudes over distances ranging from $15 R_{\odot}$ to more than $100 R_{\odot}$ since 1982 (Bourgois et al., 1985) and on a regular basis since 1990. Simultaneous observations using all three of the EISCAT antennas allow a cross-correlation analysis (Coles, 1996; Canals et al., 2002) to be used to determine the solar wind speed(s) across the line of sight.

A comprehensive upgrade was carried out to the receivers at all mainland EISCAT sites in 2001. This upgrade has improved the reliability of the receivers and improved the quality of data returned from ionospheric soundings. However the sensitivity of IPS measurements has substantially decreased since this upgrade.

There has also been a steady encroachment from mobile telephone networks into the frequency bands used by EISCAT. Hence, in 2002, EISCAT was upgraded to allow measurements within the protected astronomy frequency band of $1.4 \mathrm{GHz}$ at two sites (Wannberg et al., 2002). The complication of a transmitting system has meant that the remaining antenna, at Tromsø, has yet to be upgraded and still uses the original observing frequency of $928 \mathrm{MHz}$. Whilst it is possible to obtain a reliable estimate of the mean solar wind velocity in the line of sight from a single-site IPS observation (Manoharan and Ananthakrishnan, 1990), cross-correlation over a long baseline provides a more accurate estimate of solar wind velocities even when more than one stream is present. Hence the ability to cross-correlate different observing frequencies assumes some importance in maintaining IPS observations with all of the baselines available at EISCAT. An added advantage of this ability is the use of the EISCAT Svalbard Radar (ESR) for IPS observations (Fallows et al., 2006), further increasing the length of baseline available and with it the accuracy with which solar wind speed and direction may be determined (Breen et al., 2006).

In this paper, the probable causes of the decreased sensitivity of IPS measurements are discussed and some recent results from dual-frequency observations presented. 


\section{Interplanetary scintillation - some background the- ory}

Density fluctuations in the solar wind, which are assumed to be of turbulent origin, cause phase variations to be introduced in the radio signal from a distant source. If the rms phase difference over transverse scales equal to the Fresnel scale $R_{f}=\sqrt{\frac{\lambda z}{2 \pi}}$ are small ( $\ll 1$ radian), then the level of scattering is said to be "weak" and diffraction can be modeled with the Born approximation as a linear summation of the effects of a series of "thin screens" along the line of sight between the source and receiver. The resulting intensity fluctuations have most of their energy at scales near $R_{f}$. Since $R_{f}$ changes with distance $z$, the line of sight integration spreads the energy over a range of scales. Higher observing frequencies, sensitive to smaller density scales, which are weaker, may be used to maintain observations within a weak scattering regime as the mean solar wind density increases closer to the Sun.

The IPS temporal power spectrum under weak scattering conditions can be expressed by the following equation: (For further details, the reader is referred to, e.g. Scott et al., 1983, and references therein).

$$
\begin{gathered}
P(f)=8 \pi r_{e}^{2} \lambda^{2} \int_{0}^{\infty} \frac{2 \pi}{v_{p}(z)} \int_{-\infty}^{\infty} \sin ^{2}\left(\frac{q^{2} \lambda z}{4 \pi}\right) \\
\left|V\left(q, z, \theta_{0}(\lambda)\right)\right|^{2} P_{\phi}(q) d q_{y} d z
\end{gathered}
$$

$P_{\phi}(q)=C\left(q_{x}^{2}+\frac{q_{y}^{2}}{A R^{2}}\right)^{-\frac{\alpha}{2}} \exp -\left(\frac{q}{q_{i}}\right)^{2} R^{-4}$

where: $r_{e}$ is the classical electron radius; $\lambda$ is the observing wavelength; $C$ is a constant of proportionality; $v_{p}$ is the component of solar wind velocity perpendicular to the line of sight; $q$ is the 2-dimensional spatial wavenumber (density variations are assumed to be anisotropic with the anisotropy defined by an axial ratio, $A R)$; $z$ is the distance from Earth to the scattering screen; $V\left(q, z, \theta_{0}(\lambda)\right)$ is the visibility function of a radio source of size $\theta_{0}(\lambda) \cdot \sin ^{2}\left(\frac{q^{2} \lambda z}{4 \pi}\right)$ is the Fresnel filter which acts as a high-pass filter attenuating wavenumbers below the Fresnel frequency, $q_{f}=\sqrt{\frac{4 \pi}{\lambda z}}$. $\exp \left(-\left(\frac{q}{q_{i}}\right)^{2}\right.$ describes the dissipation and attenuates the scintillation power spectrum at wavenumbers higher than $q_{i}$.

The radio source structure is an important factor in IPS measurements. For a radio source with a Gaussian brightness distribution $\left(B(\theta)=\exp \left(\frac{-\theta^{2}}{2 \theta_{0}^{2}}\right)\right)$, the source visibility can be expressed as $\exp \left(-\left(q z \theta_{0}\right)^{2}\right)$ (e.g. Yamauchi et al., 1996). It then acts as a low-pass filter attenuating wavenumbers above $q_{s}=\frac{1}{z \theta_{0}}$. Thus the effect of source size in the IPS power spectrum is similar to that of the inner scale. This simple Gaussian model of source structure is not necessarily valid in many cases. Radio sources may have a structure which is elongated in a particular direction, or several discrete Gaussian sources may occupy the antenna beam with similar effects. The angular extent of the radio source when projected along the direction of solar wind flow is then the dominant factor.

\section{Sensitivity of IPS measurements}

The decrease in sensitivity of IPS measurements using EISCAT is best illustrated by the direct comparison of two observations made at the same stage of the solar cycle (and so showing broadly similar solar wind streams). Such a comparison is made in Fig. 1 giving the power spectra and correlation functions from two observations of the radio source J0738+177, one taken on 13 July 1995 and the other on 13 July 2006, using the same pair of antennas.

It is evident from this comparison that the power spectra from the 1995 observation are far more clearly defined in shape than the 2006 observation, and that the power levels exceed the white noise level far more in the 1995 observation. The ratio in the power spectral density between the IPS signal and the noise baseline can be shown to be proportional to $B_{R F}\left(T_{\text {source }} / T_{\text {system }}\right)^{2}$ (e.g. Fallows et al., 2002, based on W.A.Coles, private communication). The comparison clearly shows that this factor decreased substantially from $20 \mathrm{~dB}$ in 1995 to less than $10 \mathrm{~dB}$ in 2006.

The possible causes of this change were investigated in summer 2006 by W. A. Coles (University of California, San Diego) and M. Kojima (Solar-Terrestrial Environment Laboratory, Nagoya University). Their findings are summarised in the next few sub-sections.

\subsection{Interference and bandwidth limitations}

The problem of increasing interference from GSM base stations has been known for some years. As the usual operating frequency of EISCAT (around $930 \mathrm{MHz}$ ) is not - and could not be due to transmission operations - in a protected frequency band, the bandwidth available has been steadily encroached upon. In order to try and reduce the effects of this the central observing frequency has been moved several times from the original $933.5 \mathrm{MHz}$ and now lies at $928 \mathrm{MHz}$ for the main ion line receiver. However it has also been necessary for the bandwidth to be reduced from the original $30 \mathrm{MHz}$ (reduced to $15 \mathrm{MHz}$ in c1988 with the first analogue GSM operations in the $900 \mathrm{MHz}$ waveband) to just under $8 \mathrm{MHz}$ in the case of Troms $\varnothing$ and Kiruna and $1.5 \mathrm{MHz}$ in the case of Sodankylä (where the GSM problem is much more severe). This restriction on available bandwidth helped prompt the move to $1420 \mathrm{MHz}$ IPS operations at the remote sites; Troms $\varnothing$ has yet to have such an upgrade due to the complication of the transmitting system.

In their observations of summer 2006, Coles and Kojima attempted to mitigate the effects of GSM interference by 
recording samples every $100 \mu \mathrm{s}$ instead of the more usual $10 \mathrm{~ms}$. They then reduced the sampling interval to $10 \mathrm{~ms}$ by smoothing and decimating with a median smoother rather than a mean. This was successful at eliminating most of the brief, high amplitude, pulses of GSM interference. However a median filter is less statistically efficient than a mean filter, increasing the rms by approximately $15 \%$, but is a better proposition than leaving the interference to be averaged in the usual way. This approach was less successful at Sodankylä where the interference was such that the brief pulses overlap into a more continuous stream, thus providing further evidence (above the extra restriction on bandwidth) that IPS operations are only realistic with the $1420 \mathrm{MHz}$ system on this antenna.

The receiver upgrade carried out in 2001 has also reduced the recordable bandwidth. Prior to the upgrade, the full bandwidth of $15 \mathrm{MHz}$ was recordable. However the Digital Signal Processor (DSP) installed during the upgrade is limited by a (theoretical) data transfer rate of around $20 \mathrm{Mbps}$. Whilst this is more than adequate for most EISCAT operations, it places a severe constraint on the recordable bandwidth for the much higher sampling rate used for IPS measurements. At present data are measured by three 16-bit channels (from a total of six), each covering $1.67 \mathrm{MHz}$ of bandwidth. This takes the data transfer rate very close to its theoretical limit and only provides a $5.4 \mathrm{MHz}$ bandwidth, a reduction by a factor of three compared to the old system.

\subsection{System noise temperature}

Cryogenic cooling of the preamplifiers at the remote sites was removed during the system upgrade of 2001, resulting in an increased system temperature at these sites. Coles and Kojima reported that the system temperature had risen to approximately $45 \mathrm{~K}$ from $30 \mathrm{~K}$ prior to the upgrade. Since the IPS sensitivity is inversely proportional to the square of the system temperature, the lack of cooling has further reduced the sensitivity by a factor of two.

\subsection{The $1420 \mathrm{MHz}$ system}

Observing at $1420 \mathrm{MHz}$ has the principle advantage that it lies in a long-standing radio band protected for astronomical observations of the $21 \mathrm{~cm}$ Hydrogen spectral emission line. However, the antennas of EISCAT were not designed to be at their maximum efficiency at this frequency. Coles and Kojima reported that the ratio of the aperture efficiency to system temperature drops by a factor of two compared to that at $930 \mathrm{MHz}$. They suggested that, due to antenna beams having a tendency to narrow when the frequency is slightly increased, the drop may be due to an under-illumination of the dish by the $930 \mathrm{MHz}$ feed when operated at $1420 \mathrm{MHz}$. However G. Wannberg (private communication) suggested that the dish is actually over-illuminated at $1420 \mathrm{MHz}$, allowing extra ground noise into the receiver. This has been
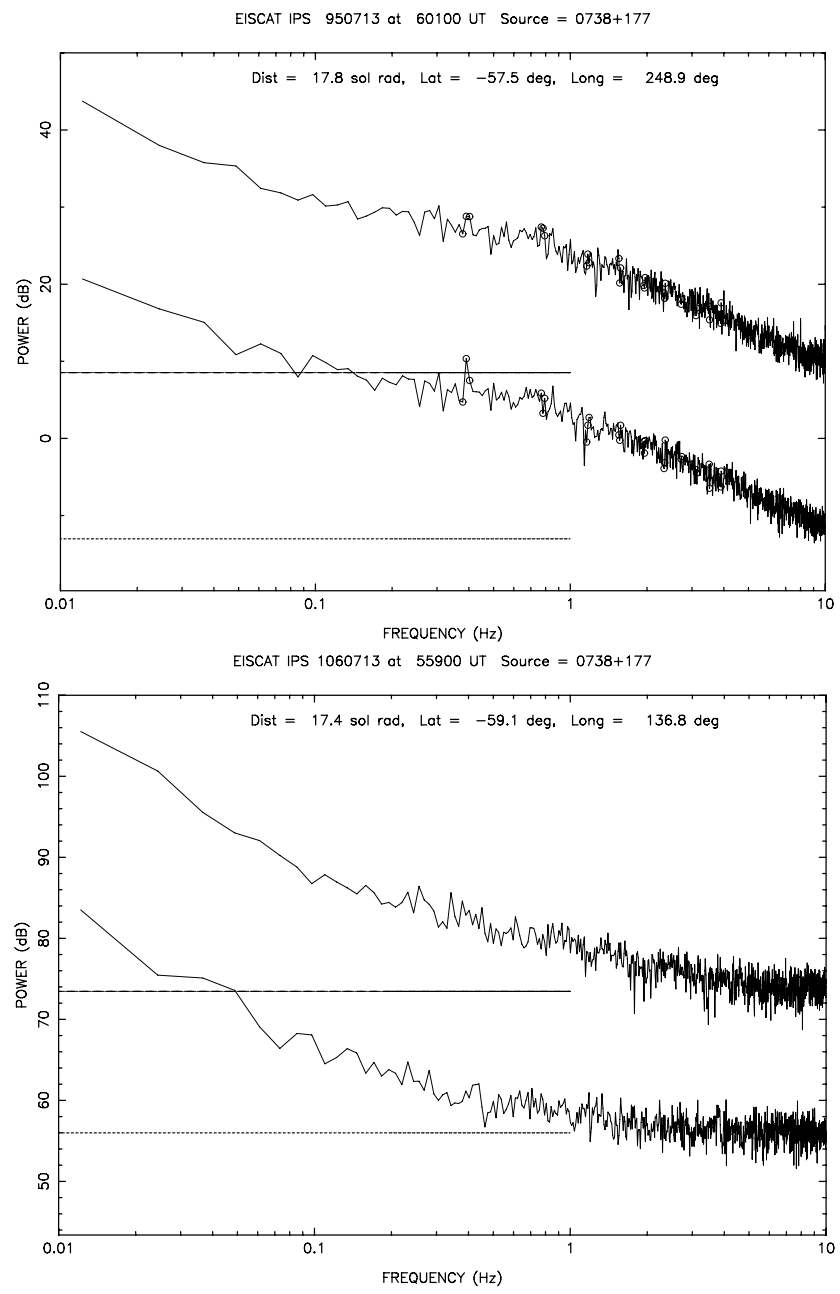

Fig. 1. Power spectra of IPS measurements of the radio source J0738+177 taken on 13 July (a) 1995 and (b) 2006. In each case the top spectrum is of data taken by the Kiruna site and the bottom spectrum is of data taken at Sodankylä. The horizontal dotted and dashed lines represent the level of background white noise for each spectrum. The power is given in decibels and the frequency displayed logarithmically.

confirmed by a set of drift scans of the strong radio sources Cas-A and Cyg-A made by EISCAT staff.

A further complication is that the radio sources themselves may be up to $50 \%$ weaker at $1420 \mathrm{MHz}$ compared with $930 \mathrm{MHz}$. The net effect is a further drop in the IPS sensitivity, but the data are not lost due to external interference.

\section{Spring 2007 trials}

Following the tests of Coles and Kojima, ways of increasing the bandwidth available for IPS with the current EISCAT system were investigated in spring 2007. These investigations 


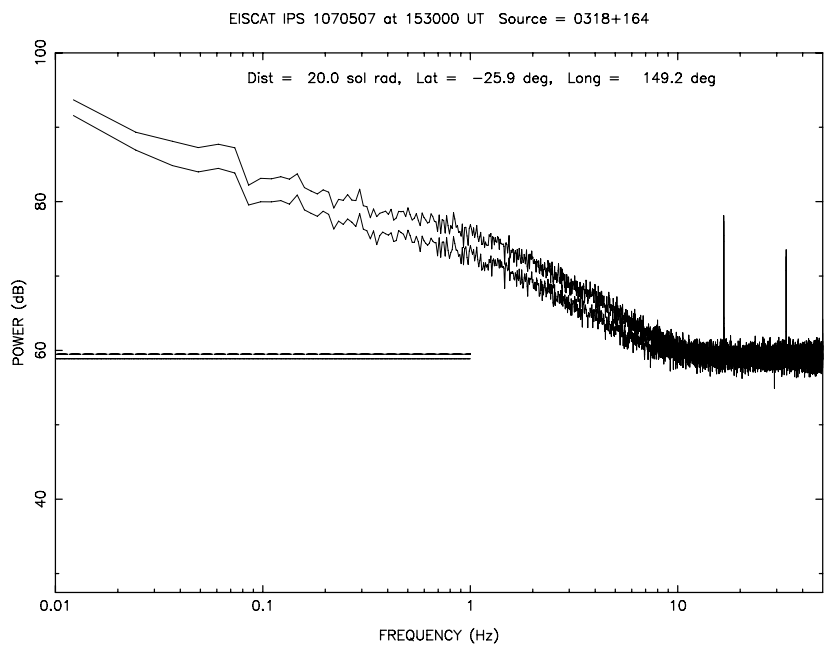

Fig. 2. Auto-power spectra of Troms $\emptyset$ data from an observation of the radio source J0318+164 taken on 5 May 2007. The plot with raised power levels in relation to the other includes data from an additional three channel boards in the VHF DSP. The horizontal dotted and dashed lines mark the background noise level for each spectrum. The power is given in decibels but is arbitrary in level; the frequency is displayed logarithmically.

met with a little success but did not result in a dramatic increase in the sensitivity.

All three sites have two signal receivers installed: at Troms $\varnothing$ the second receiver is used for plasma line measurements at a central frequency of $924 \mathrm{MHz}$; at the remote sites two polarisation channels (horizontal and vertical) are installed and the secondary receivers are used for the second polarisation. This means that the nominal $8 \mathrm{MHz}$ of potential bandwidth available can be expanded or a second orthogonal polarisation used to provide extra independent information.

However, the principle issue is the limitation on data transfer in the DSP. At Troms $\emptyset$ a second, identical DSP is used for measurements taken using the VHF receiver. It is a straightforward matter to pipe the output from the second UHF receiver to this DSP, provided the VHF antenna is not in use. This immediately doubles the $5.4 \mathrm{MHz}$ recordable bandwidth to $10.8 \mathrm{MHz}$.

There is no second DSP at the remote sites. However in conjunction with the installation of a dual polarisation system a total of eight 8-bit channel boards were built so that four of these could be used at each site as opposed to only three of the 16-bit boards. Six slots are available for channel boards in the DSP, but it is not possible to mix 8- and 16-bit boards in the same system. Hence it was decided to try and use six 8-bit channel boards at Sodankylä, leaving Kiruna with only the three 16-bit boards. The six 8-bit boards operated well at Sodankylä, thus doubling the available information here. A trial was also carried out to see if four 16-bit boards could be used: Surprisingly, this was successful at Sodankylä but did not work at Kiruna.

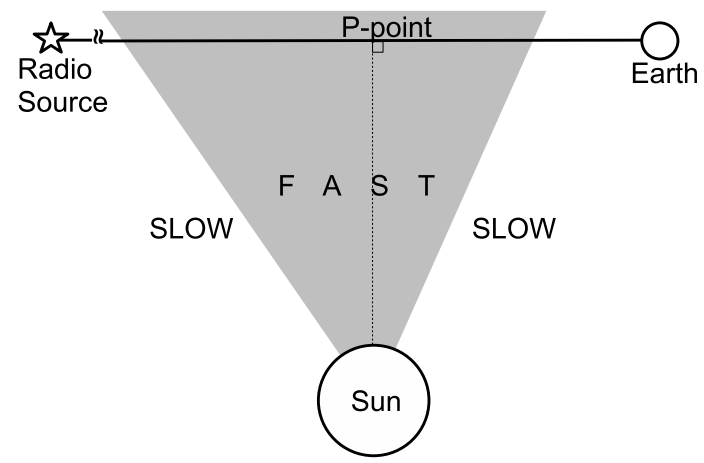

Fig. 3. A diagram illustrating the IPS line of sight as viewed from above. The point of closest approach of the line of sight to the Sun is indicated as the P-point. Example locations of fast and slow streams are given.

Figure 2 shows the auto- power spectra of Troms $\varnothing$ data from an observation of J0318+164 taken on 5 May 2007; one plot includes data from the additional three channel boards of the VHF DSP, the other does not. The difference between the two Troms $\emptyset$ spectra is readily apparent with the power levels from data including the additional three channel boards increased above the background white noise by approximately $5 \mathrm{~dB}$.

\section{A three-stream weak scattering model}

A diagram showing the IPS line of sight relative to the Sun is given in Fig. 3. When the line of sight is mapped back along the solar wind streamline to the solar corona (usually to a distance of $2.5 R_{\odot}$ ), the central portion will lie at higher solar latitudes than the tails and may be immersed in fast solar wind flow; the tails usually remain above the coronal streamer belt and so lie in slow flow. Overlaying the back-projected line of sight onto a Carrington map of coronal white light (usually taken from SoHO LASCO coronagraph data) allows the portions of the line of sight immersed in fast or slow flow to be identified reasonably accurately under straightforward solar minimum conditions (Coles, 1996).

A weak scattering interplanetary scintillation model allowing for the presence of two solar wind streams in the line of sight has been used for the last decade to analyse EISCAT IPS observations (Coles, 1996; Klinglesmith, 1997; Canals et al., 2002). The current analysis routines perform all fitting to the auto- and cross- power spectra, with the resulting correlation functions displayed for a more convenient visualisation.

In the analysis routines, the parameters $\alpha$ and the inner scale are adjusted to describe the spectrum of density variations. Previous studies (e.g. Yamauchi et al., 1996) have found $\alpha$ to vary between 2 and 4 and the inner scale in 


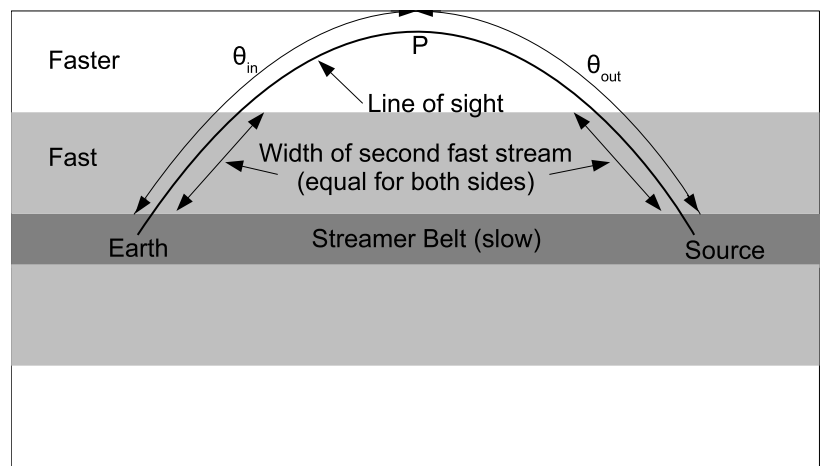

Fig. 4. Diagram demonstrating the geometry of the new model including two fast streams. The line of sight is displayed as though it were mapped back down to the corona, with the corona displayed in the form of a Carrington map. Hence the horizontal axis represents heliographic longitude and the vertical axis represents heliographic latitude with the equator across the centre. It should be noted that each stream may have a variable width, including zero.

kilometres to be roughly equal to the distance of the observation in solar radii, although it is not very well determined. These boundaries are used as a guide when matching the model to the high-frequency tail of the auto-power spectrum. Since these parameters also closely match the effect of source structure and the two effects are difficult to separate, source structure is not modelled separately in the routines and any effects are also matched with these parameters.

The density variations are assumed to be anisotropic and normally extended in the direction of the interplanetary magnetic field. The magnetic field direction is assumed to be radial but corrected to include the Parker spiral and may also be tilted in solar latitude (to include a super-radial expansion of the magnetic field for example) by an angle $\gamma$ where $\gamma_{R}=\gamma \exp \left(\frac{-R}{\gamma_{L}}\right)$ describes the decrease of this angle with distance from the Sun for points in the line of sight away from the point of closest approach to the Sun. Solar wind velocity is normally assumed to follow the direction of the magnetic field but a parameter, $\delta$, may be used to describe an angle between the two if this is thought necessary. Both of these parameters may be adjusted for each stream.

The slow stream is given an extra weighting to account for its greater density and hence greater scattering power. Following the work of Little and Ekers (1971), each stream can be modelled as having a mean speed with random velocity components in the radial $\left(\delta V_{\|}\right.$- indicated by a skewed crosscorrelation function) and transverse $\left(\delta V_{\perp}\right.$ - indicated by a reduced area under the cross-correlation function) directions.

Observations indicating the presence of two discrete fast streams in addition to a slow stream in the line of sight prompted the model to be extended to allow for the second fast stream (Bisi et al., 2007). This stream was given a slight additional weighting of 1.5 following the work of Woo and

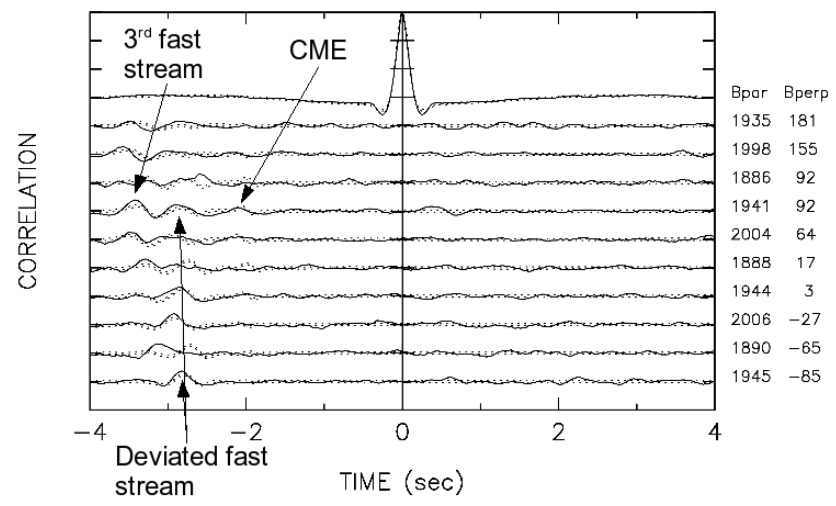

Fig. 5. Auto- (top line) and cross-correlation functions for the EISCAT-MERLIN observation of J0319+415 (3C84) after 14:10UT on 14 May 2005. The peaks associated with the perturbed background solar wind flow and with the CME are marked "Deflected fast wind" and "CME", respectively. The solid lines are the observed functions and the broken lines the weak-scattering model fit. The parallel and perpendicular baselines (Bpar and Bperp) are given in km. After Breen et al. (2008).

Habbal (1997) who noted a step in Ulysses density values, but, apart from the speed, otherwise had the same stream parameter values as the main fast stream. Figure 4 illustrates the expected line of sight geometry, displayed as though it were back-projected down to the solar corona.

However it has become apparent that some observations can only be modelled satisfactorily with full three-stream capabilities, meaning all solar wind stream related parameters being able to be individually adjusted for each stream and each stream able to be given an additional weighting (although the fast stream, being the least dense, has always had no additional weighting). Particular cases are where a Coronal Mass Ejection (CME) or, potentially, where a region of interaction between fast and slow streams is thought to exist in the line of sight. A CME observed on 14 May 2005 has been modelled this way, using a tomographic reconstruction derived from IPS observations taken with the STELab network on 13-16 May 2005 (using the method described in Jackson et al., 1998, and Jackson and Hick, 2005) to constrain the location of the CME in the line of sight (Breen et al., 2008). The modelled cross-correlation functions for a number of antenna baselines is given in Fig. 5. In this case the model (given the constraints of the tomographic analysis) was able to clearly identify a low, small-time-lag peak (previously disregarded) with the CME and indicate that the adjacent fast stream appeared to be deviated from the radial direction by approximately $15^{\circ}$.

The three-stream model is very flexible but has a large number of free parameters. It should be noted that the stream parameters are only used if the observation can support them or additional constraints can be put upon them. The 


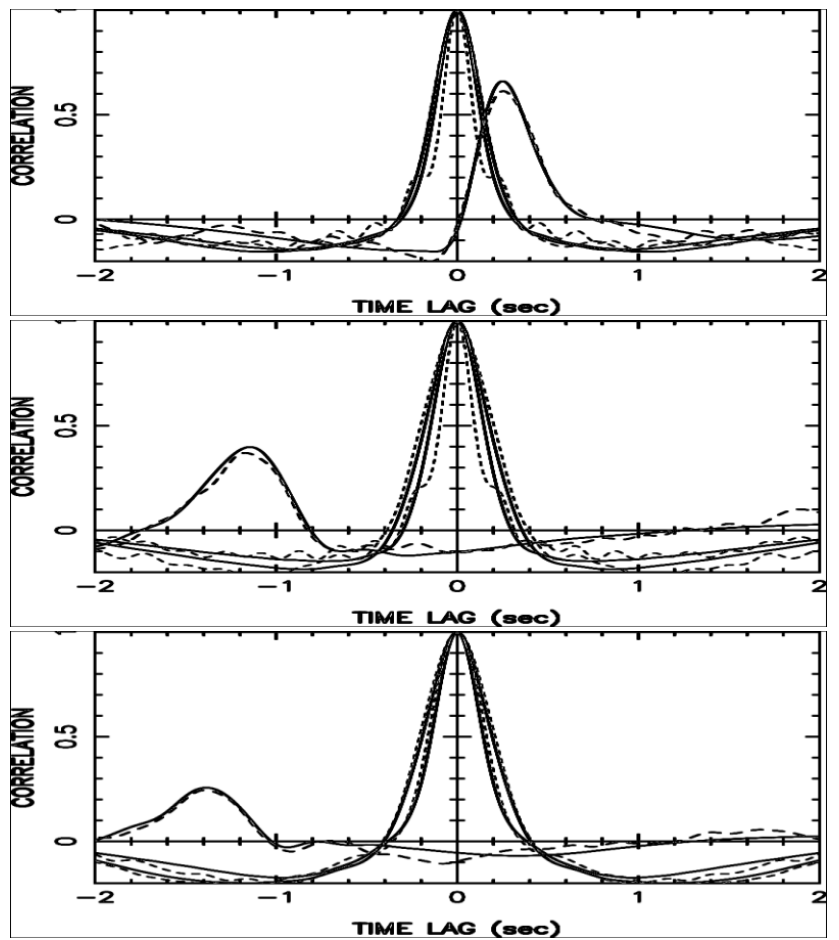

Fig. 6. An observation of the radio source J0319+415 taken on 12 May 2004, fitted using a dual-frequency weak scattering model. The top panel is a Troms $\varnothing(928 \mathrm{MHz})-$ Kiruna $(1420 \mathrm{MHz})$ correlation; the middle panel is a Troms $\varnothing(928 \mathrm{MHz})-\mathrm{ESR}(500 \mathrm{MHz})$ correlation; the bottom panel is a Kiruna (1420 MHz) - ESR $(500 \mathrm{MHz})$ correlation. The dotted and dashed lines represent the data; the solid lines the model fit. The auto-correlation functions from both antennas and the cross-correlation function are displayed in each case. The observation is dominated by fast solar wind to which the model fitted a speed of $775 \mathrm{~km} \mathrm{~s}^{-1}$.

cross-correlation peak naturally constrains the solar wind speed; $\delta V_{\perp}$ is rarely used but may be necessary for observations close to the Sun where Alfven waves become important; $\delta V_{\|}$is only required for short-baseline observations where separate streams simply skew the cross-correlation function - in longer baselines streams separate into seperate cross-correlation peaks as the difference in time lag between them increases; $\gamma$ and $\delta$ can only be used in observations where a number of cross-correlation functions covering a range of baselines exist and these indicate that the flow may not be purely radial.

\section{Dual-frequency observations}

The principle science from EISCAT IPS observations has come from the cross-correlation of measurements from very widely-spaced antennas (e.g. Bisi et al., 2007; Breen et al., 2006). As it has become necessary to operate the Sodankylä antenna at $1420 \mathrm{MHz}$, but Troms $\emptyset$ has yet to be converted to this frequency, dual frequency correlation is necessary in order to maintain use of the longest baseline available between the mainland EISCAT sites. Following successful dual-frequency trials in 2003, use of the EISCAT Svalbard Radar for IPS was started in 2004, enabling very long baselines (>1000 km) to be used (Fallows et al., 2006).

Equation (1) assumes a single observing frequency and so can only represent the auto-spectrum for each antenna in the case of dual-frequency observations. A simple modification is needed for the cross-spectrum. Salpeter (1967) is one of few authors on IPS theory to do this: All the $\lambda^{2}$ terms are separated into single $\lambda$ terms and the cross-spectrum becomes:

$$
\begin{aligned}
P(f)= & 8 \pi^{2} r_{e}^{2} \lambda_{1} \lambda_{2} \int_{0}^{\infty} \frac{2 \pi}{v_{p}(z)} \\
& \int_{-\infty}^{\infty} \sin \left(\frac{q^{2} \lambda_{1} z}{4 \pi}\right) \sin \left(\frac{q^{2} \lambda_{2} z}{4 \pi}\right) \\
& \left|V\left(q, z, \theta_{0}\left(\lambda_{1}\right)\right)\right|\left|V\left(q, z, \theta_{0}\left(\lambda_{2}\right)\right)\right| P_{\phi} d q_{y} d z
\end{aligned}
$$

The assumption of weak scatter must be valid for both observing frequencies. The cross correlation between different frequencies drops much more quickly in strong scattering than is the case for single-frequency cross-correlation, so this technique cannot be used as close to the Sun as the single frequency case. By contrast, any radio source is limited in how far from the Sun its scintillation can be measured at a given frequency by its strength, angular size and structure. Therefore radio sources and heliocentric distances have to be picked carefully to ensure that these restrictions are satisfied for both observing frequencies.

The radio source visibility function has also been separated into two terms, each dependent on an observing frequency, to account for differing radio source structures which may be apparent at each frequency.

The weak-scattering model currently in use is restricted to using a single observing frequency only, although it has been found that using an average of the two frequencies in a dual frequency observation seems to give reliable results. A full dual-frequency model based on Eq. (3) is under development and has now reached the testing stage. Figure 6 gives an example fit using this model to an observation of J0319+415 made on 12 May 2004. It can be seen that the model fits the correlation functions in all three cases very well, giving a solar wind speed of $775 \mathrm{~km} \mathrm{~s}^{-1}$.

The correlation functions given in Fig. 6 were fitted using single $\alpha$ and inner scale model parameters, despite the radio source structure at each frequency being included in them. In this case the fits are still good but the final model will separate these parameters according to observing frequency, with the cross-correlation model using an average of the two, to allow differing source structures to be accounted for.

Tests using the new model are still continuing, but it is expected to go into full-time use in the near future. 


\section{Summary and conclusions}

Coles and Kojima carried out a comprehensive investigation into the loss of sensitivity of the EISCAT system for IPS, apparent between measurements carried out in the mid-1990s and 2006. Their findings indicated that a number of factors are to blame. The principle factor is the lack of recordable bandwidth forced by GSM base stations encroaching on the frequencies used by EISCAT and also by the data transfer limit imposed by the Digital Signal Processors installed during the 2001 system upgrade.

The interference due to GSM signals could be mitigated at Troms $\varnothing$ and Kiruna by smoothing and decimating the data samples to the required time interval using a median smoother. However the inteference was too great at Sodankylä for this method to be useful there, rendering IPS observations at $928 \mathrm{MHz}$ impractical at this site.

The $1420 \mathrm{MHz}$ upgrade enables observations in a protected frequency band. Since the upgrade was only carried out at the remote sites, it has proved necessary to crosscorrelate measurements taken at two different observing frequencies in order to maintain the length of baselines most useful for the science being carried out. This in turn has enabled use of the ESR for IPS measurements providing very long baselines with the associated improvement in the ability to resolve different solar wind streams in the line of sight and improved sensitivity to solar wind flow direction.

The weak-scattering model used to analyse IPS measurements has been extended to allow for a third stream in the line of sight, improving the accuracy with which CME observations, in particular, may be modelled. A full dual-frequency extension is currently being tested and expected to go into full service in the near future. Initial results using this model are very encouraging.

Trials were carried out in spring 2007 to investigate how the bandwidth could be increased within the limits of the current system. It was found to be possible to double the bandwidth at Troms $\varnothing$ through use of the additional VHF receiver DSP and at one of the remote sites by using six 8-bit channel boards. A small increase in sensitivity was noted.

The current system is excellent for the uses (mostly ionospheric studies) for which it was intended, but has had a substantial impact on IPS observations. In order to fully restore the sensitivity to IPS that EISCAT had prior to the 2001 upgrade, further upgrades are necessary. Coles and Kojima proposed, as a temporary solution, the use of seperate analogue square-law detectors and settings within the DSP that allow the detected signal power to be recorded directly to disk. They proposed the installation of a filterbank backend, such as is used in several radio astronomy observatories, to cover a much wider bandwidth with efficient interference rejection. The Aberystwyth University IPS group is currently investigating ways of increasing the available bandwidth. The installation of low-maintenance cooling systems at the remote sites would also reduce the noise floor, to the benefit of the whole EISCAT user community.

Acknowledgements. We would like to extend particular thanks to W. A. Coles and M. Kojima for their thorough investigation into the IPS sensitivity and for making their report available to us. We would also like to thank W. A. Coles and B. Rickett for making their original IPS analysis routines available to us. R. A. Fallows, A. R. Breen and G. D. Dorrian were supported by the UK's STFC/PPARC during the time this work was carried out. EISCAT is supported by the research councils of Norway, Sweden, Finland, Japan, China, the United Kingdom and Germany.

Topical Editor K. Kauristie thanks W. Coles and G. Wannberg for their help in evaluating this paper.

\section{References}

Bisi, M., Fallows, R., Breen, A., Habbal, S., and Jones, R.: LargeScale Structure of the Fast Solar Wind, J. Geophys. Res., 112, A06101, doi:10.1029/2006JA012166, 2007.

Bourgois, G., Coles, W., Daigne, G., Silen, J., Turunen, T., and Williams, P.: Measurements of the Solar Wind Velocity with EISCAT, Astron. Astrophys, 144, 452, 1985.

Breen, A., Coles, W., Grall, R., Klinglesmith, M., Markkanen, J., Moran, P., Tegid, B., and Williams, P.: EISCAT Measurements of the Solar Wind, Ann. Geophys., 14, 1235-1245, 1996b, http://www.ann-geophys.net/14/1235/1996/.

Breen, A., Fallows, R., Bisi, M., Thomasson, P., Jordan, C., Wannberg, G., and Jones, R.: Extremely Long Baseline Interplanetary Scintillation Measurements of Solar Wind Velocity, J. Geophys. Res., 111, A08104, doi:10.1029/2005JA011485, 2006.

Breen, A., Fallows, R., Bisi, M., Jones, R., Jackson, B., Kojima, M., Dorrian, G., Middleton, H., Thomasson, P., and Wannberg, G.: The Solar Eruption of 13 May 2005 and its Effects: LongBaseline Interplanetary Scintillation Observations of the EarthDirected Coronal Mass Ejection, Astrophys. J. Lett., 683, L79, in press, 2008.

Canals, A., Breen, A., Moran, P., and Ofman, L.: Estimating Random Transverse Velocities in the Fast Solar Wind from EISCAT Interplanetary Scintillation Measurements, Ann. Geophys., 20, 1265-1277, 2002, http://www.ann-geophys.net/20/1265/2002/.

Coles, W.: Interplanetary Scintillation, Space Sci. Rev., 21, 411425, 1978 .

Coles, W.: A Bimodal Model of the Solar Wind Speed, Astrophys. Space Sci., 243, 87-96, 1996.

Fallows, R., Williams, P., and Breen, A.: EISCAT Measurements of Solar Wind Velocity and the Associated Level of Interplanetary Scintillation, Ann. Geophys., 20, 1278-1290, 2002, http://www.ann-geophys.net/20/1278/2002/.

Fallows, R., Breen, A., Bisi, M., and Jones, R.: Dual-Frequency Interplanetary Scintillation Observations of the Solar Wind, Geophys. Res. Lett., 33, L11 106, doi:10.1029/2006GL025804, 2006.

Hewish, A., Scott, P., and Wills, D.: Interplanetary Scintillation of Small Diameter Radio Sources, Nature, 203, 1214-1217, 1964.

Jackson, B. and Hick, P.: in: Solar and Space Weather Radiophysics, edited by: Gary, D. and Keller, C., vol. 314 of Astrophys. and Space Sci. Lib., p. 355, Kluwer, 2005. 
Jackson, B., Hick, P., Kojima, M., and Yokobe, A.: Heliospheric Tomography Using Interplanetary Scintillation Observations: 1. Combined Nagoya and Cambridge Data, J. Geophys. Res., 103, 12 049-12 067, 1998.

Klinglesmith, M.: The Polar Solar Wind from 2.5 to 40 Solar Radii: Results of Intensity Scintillation Measurements, Ph.D. thesis, University of California, San Diego, 1997.

Little, L. and Ekers, R.: Method for Analysing Drifting Random Patterns in Astronomy and Geophysics, Astron. Astrophys, 10, 306-309, 1971.

Manoharan, P. and Ananthakrishnan, S.: Determination of Solar Wind Velocities Using Single-Station Measurements of Interplanetary Scintillation, Mon. Not. R. Astr. Soc., 244, 690-695, 1990.

Salpeter, E.: Interplanetary Scintillations I. Theory, Astrophys. J., 147, 433-448, 1967.
Scott, S., Rickett, B., and Armstrong, J.: The Velocity and the Density Spectrum of the Solar Wind From Simultaneous ThreeFrequency IPS Observations, Astron. Astrophys, 123, 191-206, 1983.

Wannberg, G., Vanhainen, L.-G., Westman, A., Breen, A., and Williams, P.: The new $1420 \mathrm{MHz}$ dual-polarisation interplanetary scintillation (IPS) facility at EISCAT, in: Proc. Union of Radio Scientists (URSI) 2002, 2002.

Woo, R. and Habbal, S.: Extension of Coronal Structure into Interplanetary Space, Geophys. Res. Lett., 24, 1159-1162, 1997.

Yamauchi, Y., Kojima, M., Tokumaru, M., Misawa, H., Mori, H., Tanaka, T., Takaba, H., Kondo, T., and Manoharan, P.: MicroTurbulence in the Solar Wind at 5-76 Rs Observed with Interplanetary Scintillation, J. Geomag. Geoelectr., 48, 1201-1217, 1996. 